\title{
Tipologie amministrative della signoria rurale in Italia tra medioevo ed età moderna
}

\author{
di Sandro Carocci
}

Lamministrazione signorile rappresenta un tema immenso, pochissimo trattato dalla storiografia e comunque mai oggetto di ricostruzioni complessive. L'articolo fornisce un primo quadro d'insieme, del tutto provvisorio, di forme di gestione diverse a seconda delle epoche, delle regioni, del tipo di signoria, delle sue entrate e di molti altri fattori. Ricorre alla nozione weberiana di idealtipo per cercare di dominare la molteplicità e la varietà delle forme di amministrazione concretamente utilizzate, individuando i due idealtipi di base "Gestione diretta" e "Gestione delegata", a loro volta suddivisi in cinque sotto idealtipi. Si interroga infine sulle ricadute economiche e sociali di ciascuna forma di gestione, e sulla possibilità di individuare, a seconda delle epoche e delle regioni, una diffusione più o meno ampia di signorie riconducibili ai diversi idealtipi.

Lordship management is a broad subject scarcely treated by historians; even when analysed, it has never been the object of exhaustive reconstructions and systematic surveys. The article provides a first, provisional overview of the main forms of lordship administration that changed depending on the period, region, lordship, type of revenue and many other factors. The Weberian notion of ideal type is particularly helpful in order to try to master the multiplicity and variety of forms of administration used in practice. On its basis, the essay identifies two basic ideal types, 'Direct Management' and 'Indirect Management', which are in turn divided into five sub-ideal types. Lastly, it questions the economic and social effects of each form of management, and the possibility of identifying, depending on the period and region, a more or less extensive diffusion of lordships attributable to the different ideal types.

Medioevo; età moderna; Italia; signoria rurale; idealtipo di Max Weber.

Middle Ages; Modern era; Italy; lordship; Max Weber Ideal type.

Sandro Carocci, University of Rome Tor Vergata, Italy, carocci@lettere.uniroma2.it, 0000-0001-9989-6780

FUP Best Practice in Scholarly Publishing (DOI 10.36253/fup_best_practice)

Sandro Carocci, Tipologie amministrative della signoria rurale in Italia tra medioevo ed età moderna, pp. 19-39, () 2021 Author(s), content CC BY 4.0 International, DOI 10.36253/978-88-5518-423-6.02, in Paola Guglielmotti, Isabella Lazzarini (edited by), "Fiere vicende dell'età di mezzo». Studi per Gian Maria Varanini, (C) 2021 Author(s), content CC BY 4.0 International, metadata CC0 1.0 Universal, published by Firenze University Press (www.fupress.com), ISSN 2704-6079 (online), ISBN 978-88-5518-423-6 (PDF), DOI 10.36253/978-88-5518-423-6 
Gli studi su singole signorie offrono indicazioni, talvolta preziose, sui modi con cui uomini, terre, rendite e diritti venivano sorvegliati e valorizzati economicamente dai signori. In Italia, però, solo in pochi casi le forme gestionali delle signorie medievali sono state oggetto di analisi specifiche e approfondite, al contrario di quanto è accaduto per l'età moderna, che vanta ricerche molto ricche di dati e di osservazioni. Quest'assetto degli studi rende particolarmente azzardato il tentativo proposto in questo contributo: elaborare una tipologia delle forme di gestione delle signorie rurali che affiorano negli studi condotti in tutte le regioni italiane, dalle Alpi alla Sicilia, e su un lungo arco cronologico, dal XII secolo all'età moderna. A mia discolpa, posso invocare due elementi. Da un lato, i risultati del Progetto di Rilevante Interesse Nazionale, La signoria rurale nel XIV-XV secolo: per ripensare l'Italia tardomedievale, a cui Gian Maria Varanini ha generosamente partecipato come autore di saggi importanti e come organizzatore di un'intera sezione regionale ${ }^{2}$. Dall'altro lato, un incontro di ricerca con studiosi della fiscalità signorile in Spagna, dove gli organizzatori mi hanno chiesto di trattare le forme di amministrazione della signoria in Italia in modo generale, al fine di agevolare una comparazione con la realtà iberica ${ }^{3}$. Le pagine seguenti sono una rielaborazione del testo preparato per quell'occasione, e forniscono dunque un quadro d'insieme solo provvisorio e solo molto parziale ${ }^{4}$. Devono essere intese come un invito alle ricerche, non come la sistematizzazione di conoscenze acclarate.

Nel medioevo, le signorie rurali erano presenti in tutta Italia, ma a seconda delle regioni e delle epoche cambiavano molto sia il tipo di signore più frequente, sia la percentuale di abitanti e terre della regione che i signori controllavano ${ }^{5}$. In alcune regioni la signoria è stata un elemento basilare degli assetti locali a partire dal XII secolo, al più tardi, e fino all'età moderna. Una centralità della signoria nell'organizzazione della società e del potere in realtà caratterizzava nel XII secolo gran parte dell'Italia, salvo le aree più vicine alle città e sporadiche eccezioni regionali e sub-regionali, come il territorio di Pisa, alcune aree alpine, il Friuli, la Sardegna e la Sicilia. Nel tardo medioevo e in età moderna la situazione appare mutata. In alcune regioni, la diffusione massiccia della signoria avvenne proprio nel XIV secolo: è il caso di Friuli, Sicilia e per certi aspetti anche Sardegna. In altre aree, viceversa, la signoria

\footnotetext{
${ }^{1}$ I risultati sono raccolti in La signoria rurale, a cura di Gamberini e Pagnoni; La signoria rurale, a cura di Senatore; La signoria rurale, a cura di Fiore e Provero; La signoria rurale, a cura di Carocci; La signoria rurale, a cura di Del Tredici.

2 Le signorie trentine, a cura di Varanini.

3 Si tratta del "Coloquio internacional" Gobierno y gestión de las haciendas y economías señoriales en la Europa Occidental (siglo XIII-XVI), organizzato da presso l'Universidad de Málaga, il 4-5 novembre 2019, da Ángel Galán Sánchez e Jesús Manuel García Ayoso, che ringrazio cordialmente.

4 Quadri della ricerca fino a tutto il XIII secolo sono Carocci, Signoria, prelievo rurale, e Provero, Forty Years; per i secoli XIV-XV si vedano i volumi citati sopra alle note 1 e 2.

5 Quadri regionali sono presentati per i secoli XI-XIII in Strutture e trasformazioni della signoria, a cura di Dilcher e Violante; La signoria rurale nel medioevo italiano, a cura di Spicciani e Violante; per i secoli XIV-XV si può ora contare su La signoria rurale, a cura di Del Tredici.
} 
era molto diminuita di importanza e diffusione già nel corso del XIII secolo. Negli ultimi due secoli del medioevo, la signoria aveva un ruolo importante nel definire poteri, società e economia in Sardegna, Piemonte-Valle d'Aosta, Liguria interna, Trentino, Veneto alpino e pianura padana ad est del fiume Piave, in molte ma non tutte le aree appenniniche, in singole zone della Toscana meridionale e dell'Umbria sud-occidentale, nel Lazio, e in molte regioni del mezzogiorno, Sicilia inclusa. In altre regioni o aree sub-regionali osserviamo invece, al chiudersi del medioevo, quella che possiamo definire una marginalità signorile, cioè l'assenza o la scarsa rilevanza dei poteri, dei possessi e dei prelievi signorili nel definire gli assetti locali. Le zone che possiamo chiamare a marginalità signorile erano Alpi lombarde, Pianura Padana a oriente di Vercelli, la maggior parte di Toscana e Umbria, le Marche centro-meridionali, alcune aree appenniniche (come la provincia papale di Spoleto), e i dintorni di alcune grandi città meridionali, come L'Aquila, Bari e Napoli.

A complicare questa sommaria geografia regionale va aggiunto che, fra XII secolo ed età moderna, la definizione di signoria rurale si applica a soggetti e a rapporti di potere diversissimi: dal grande nobile che possedeva decine di villaggi e migliaia di sottoposti al semplice cavaliere dotato solo di pochi contadini dipendenti. È ancora presto per dire come la tipologia dei signori sia andata cambiando durante il lungo periodo qui considerato. La crisi delle signorie episcopali e monastiche esercitate in modo diretto, e non tramite la semplice cessione in feudo dei possessi, appare nei secoli XIV-XV un elemento presente in quasi tutte le regioni, pur se vi sono celebri eccezioni, come l'abbazia di San Giusto di Susa, il capitolo cattedrale di Trento, i possessi del vescovo di Siena a sud della città e di alcuni istituti religiosi di Roma, e poi le signorie di antica e meno antica origine di alcuni monasteri meridionali, come Montecassino e Cava ${ }^{6}$. In ambito laico, le tendenze sono meno uniformi. Se si guarda alle dimensioni dei dominati, in tutto il meridione sembra di osservare una contrazione radicale delle signorie a piccola o piccolissima scala, che dominavano fra i dieci e i cento contadini soltanto e che erano molto diffuse fino alla metà del XIII secolo; dal pieno XIV secolo appare poi chiara la netta espansione dei dominii nelle mani di alcune grandi stirpi. Sempre dal punto di vista delle dimensioni, nelle altre zone d'Italia l'evoluzione è meno uniforme. Nel Piemonte sud-orientale e nel Vercellese, in Sardegna, nel Lazio e in alcune delle altre zone dove la signoria sopravvisse come un elemento cruciale di organizzazione sociale e politica, le signorie per così dire puntuali, estese su un singolo villaggio, andarono diminuendo, mentre più frequente appare la costituzione di grandi reti parentali a volte in possesso di decine di castelli. Ma in altre aree (come ad esempio il territorio ad occidente di Torino) le signorie monocastellane resistettero e si moltiplicarono.

${ }^{6}$ Orla, Abbazia di San Giusto di Susa; Curzel, Capitolo della cattedrale di Trento; Paganelli, Signoria del vescovo di Siena; Lattanzio, Signoria dell'abbazia di S. Paolo fuori le mura di Roma; Berardozzi, La signoria dell'ospedale di Santo Spirito in Sassia di Roma; Morra, Santissima Trinità di Cava de' Tirreni. 
$\mathrm{Al}$ di là delle difficoltà a ripercorrere l'evoluzione nel tempo della tipologia signorile nelle varie regioni, deve comunque essere ricordato un elemento, che è quello più importante in questa sede: signorie così diverse e così diversamente presenti nel territorio italiano a seconda delle varie epoche utilizzavano forme di amministrazione diversissime. Le cose cambiavano moltissimo a seconda delle epoche, delle regioni, della tipologia di signoria, e - quel che è più grave per chi deve cercare una sintesi - da signoria a signoria, in base a mille fattori locali, e persino all'interno di una medesima signoria, visto che per le singole entrate il signore poteva adottare forme di gestione diverse.

Se vogliamo cercare di dominare la molteplicità e la varietà delle forme di amministrazione utilizzate dalle tante e così diversificate signorie rurali italiane è utile ricorrere alla nozione weberiana di idealtipo. Come vedremo subito, ho pensato che si possono distinguere due idealtipi di base, a loro volta suddivisi in cinque sotto idealtipi.

Prima di illustrarli, devo ricordare un importante punto di metodo. Come aveva sottolineato Max Weber, lo scopo delle classificazioni idealtipiche non è quello di descrivere la realtà, ma di individuare dei modelli di riferimento a cui paragonare la realtà stessa. Gli idealtipi sono modelli astratti, che servono per interpretare la realtà concreta. La realtà è molto più complicata delle classificazioni idealtipiche ${ }^{7}$. A volte una forma di gestione poteva stare a cavallo fra due idealtipi; spesso, poi, un signore utilizzava contemporaneamente forme di gestione che rientrano in più di un idealtipo. Ciò non toglie che elaborare idealtipi aiuta a cogliere le differenze e permette di orientarsi nell'infinita varietà delle concrete forme di amministrazione signorile.

I due idealtipi di base e i cinque sotto idealtipi sono riassunti nello schema seguente:

\section{Due idealtipi base - cinque sotto idealtipi}

A. Gestione diretta: l'amministrazione di una signoria è controllata direttamente dal signore.

A1 Gestione diretta praticata personalmente dal signore.

A2. Gestione diretta realizzata tramite ufficiali.

B. Gestione delegata: assenza di partecipazione diretta del signore all'amministrazione, di persona o tramite ufficiali strettamente controllati.

B1. Gestione delegata al notabilato locale.

B2. Gestione delegata a professionisti esterni.

B3. Gestione assente.

7 «Un tipo ideale è ottenuto attraverso l'accentuazione unilaterale di uno o di alcuni punti di vista, e attraverso la riunione di una quantità di fenomeni particolari diffusi e discreti, esistenti qui in maggiore e là in minore misura, e talvolta anche assenti, in un quadro concettuale in sé unitario. Considerato nella sua purezza concettuale, questo quadro non può mai essere rintrac-

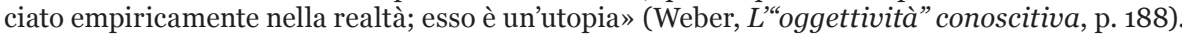


Tipologie amministrative della signoria rurale in Italia tra medioevo ed età moderna

\section{La gestione diretta praticata personalmente dal signore (A1)}

Un grande numero di signori non si occupava della amministrazione corrente dei propri dominii, o tutt'al più controllava in modo diretto soltanto un singolo aspetto della signoria (di solito, l'amministrazione della giustizia). Altri signori, invece, facevano la scelta opposta, controllando direttamente tutta o gran parte della gestione. Possiamo allora ricondurli all'idealtipo di base A, vale a dire Gestione diretta. La scelta di partecipare in modo diretto all'amministrazione poteva avere diverse motivazioni. A volte dipendeva dalla modesta estensione delle terre e dal piccolo numero di uomini dominati, che facilitavano il controllo e rendevano importante ridurre al minimo le spese di gestione. Altre volte era una scelta economica, che mirava a valorizzare al massimo le risorse della signoria. Sempre, comunque, rinvia a modelli culturali e sociali che ammettevano, e anzi a volte imponevano l'interessamento diretto di chi dominava circa le modalità di gestione delle terre, degli immobili e degli uomini a lui sottoposti. Questo interessamento e questa partecipazione potevano assumere forme diverse, che possiamo articolare in due sotto idealtipi.

A volte il signore si dedicava di persona alla gestione signorile, e possiamo allora ricondurlo al sotto idealtipo A1, gestione diretta praticata personalmente dal signore. Questi signori si interessavano di persona a tutta o quasi tutta l'amministrazione della signoria. Amministravano la giustizia, sorvegliavano la manutenzione delle fortificazioni, si preoccupavano della coltivazione delle riserve, della riparazione mulini, della puntuale riscossione di imposte e canoni.

Nei secoli XIII-XVI non era la forma di amministrazione più diffusa. Compare con frequenza solo nelle piccole signorie che, nel Sud d'Italia e in alcune altre regioni, appartenevano a cavalieri ${ }^{8}$. Per signori di maggiore livello sembra un'eccezione, magari dovuta a una contingenza particolare. Ad esempio Giovanni Antonio, uno dei rampolli del vasto consorzio dei marchesi del Carretto, ben difficilmente avrebbe presenziato di persona ai lavori di fienagione e di macinatura del grano al mulino se non fosse stato relegato dall'ostilità del padre nella piccola signoria di Levice ${ }^{9}$. È anche il tipo di amministrazione meno conosciuto, perché è quello che dava luogo alla più bassa produzione di fonti scritte. Ci è noto indirettamente, soprattutto tramite sentenze e conflitti giudiziari, e da poche fonti dirette.

Le fonti migliori sono gli inventari e le ricognizioni che questi signori potevano effettuare a scopi di controllo. Un esempio remoto è un documento fatto redigere nel 1182 da un cavaliere di Sorrento, Rainone, per la sua signoria nelle campagne di Maddaloni, a nord di Napoli: elenca tutti i beni concessi a tredici contadini che sono sottoposti personali di Rainone, soggetti alla sua

8 Carocci, Signorie di Mezzogiorno, pp. 265-28o.

9 Musso, I del Carretto, p. 51. 
giustizia e obbligati a versare censi in denaro e natura, pagare alcune imposte e fornire un numero elevato di corvées, anche oltre il centinaio ${ }^{10}$. Quarant'anni più tardi, una sentenza relativa ai cavalieri della città da cui viene Rainone, Sorrento, attesta che a decine avevano ognuno un piccolo gruppo di contadini con le relative terre, e che richiedevano un elevato numero di corvées (in questo caso oltre 110) per coltivare le loro vigne ${ }^{11}$. Possiamo credere che la maggioranza di questi piccoli signori sorvegliassero direttamente i loro sottoposti.

La partecipazione di persona alla gestione non era però una prerogativa di questi piccoli signori. Alla fine del XIV secolo e nella prima metà del successivo, in Friuli i superstiti registri di censi, affitti e spese (i cosiddetti rotuli) risultano in molti casi compilati di prima mano da personaggi di grande rilievo come i conti di Strassoldo e i signori del castello di Colloredo. Giorno dopo giorno, costoro si affaticano ad annotare i censi dovuti e quelli effettivamente riscossi, aggiungendo molteplici altre notizie gestionali. «Redazioni personali e dirette, questi registri illustrano un rapporto con i contadini che non conosceva una intermediazione organica, nella forma cioè di un fattore generale $o$ comunque di qualcuno che potesse ricavare un suo margine di profitto da un lavoro di gestione e di amministrazione» svolto per conto dei signori ${ }^{12}$.

Ciò non toglie che i signori avessero quasi sempre bisogno di aiuti. Ne potevano fare a meno, forse, quei cavalieri dotati di tre o quattro dipendenti che compaiono in alcune fonti dell'Italia meridionale. Ma se la signoria era meno lillipuziana, qualche aiuto serviva. I nobili del Friuli dalle mani sporche di inchiostro non potevano efficacemente riscuotere quei censi che registravano minuziosamente senza l'aiuto dei decani, cioè di contadini agiati che avevano il duplice ruolo di capi villaggio e di responsabili dei diritti dei signori ${ }^{13}$.

Il caso dei signori friulani costituisce un primo esempio di una complicazione che ci si porrà più volte, perché come ho avvertito è un problema ricorrente quando si paragona la concreta realtà storica con le astrazioni idealtipiche. Questa complicazione è rappresentata dalla compresenza di più idealtipi. I decani del Friuli non impediscono di parlare di una gestione praticata in modo diretto e di persona dal signore (sotto idealtipo A1), ma ci portano anche al secondo sotto idealtipo.

\section{La gestione diretta realizzata tramite ufficiali (A2)}

Rientrano in questo sotto idealtipo i signori che amministrano in modo diretto la signoria utilizzando degli aiutanti sui quali mantengono un con-

\footnotetext{
10 Giorgi, Confessione di vassallaggio.

11 Carocci, Signorie di Mezzogiorno, pp. 274-278.

12 Magistrale analisi dei rotuli friulani e della loro storia è Le campagne friulane, a cura di Cammarosano (la citazione nel testo a p. 72).

13 Ibidem, pp. 71-75.
} 
trollo efficace, a volte persino stretto. Questo controllo è garantito dalla breve durata degli incarichi o comunque dalla possibilità di porvi fine a piacimento del signore, e si accompagna all'obbligo di consultare il signore e a fornirgli rendicontazioni periodiche.

Una grande varietà di forme di amministrazione può essere ricondotta a questo sotto idealtipo. Inizierò con un esempio tardo, quello del potentissimo Cicco Simonetta, segretario del duca di Milano dal 1451 al 1479. Nella cittadina di Sartirana e in quattro villaggi vicini Cicco creò una signoria basata su un controllo attento di terre e uomini, allo scopo di massimizzare i profitti ritraibili da località situate nella fertile bassa pianura lombarda. Sartirana era un centro popoloso e socialmente articolato, con proprietari fondiari, mercanti, professionisti, nobili, e da tempo organizzato in comune. Un controllo totale era impossibile. Eppure Cicco si dotò in pochi anni di strumenti formidabili di intervento, che utilizzava e dirigeva da Milano, dove risiedeva: il governo locale fu affidato a un podestà, che consultava il signore per ogni questione e era sottoposto a controlli continui; la giustizia era amministrata, fino alla pena di morte, da giudici scelti da Cicco e a lui legati. Vi erano poi una serie di ufficiali locali minori, e un gran numero di personaggi forestieri prossimi al signore fatti trasferire a Sartirana. Cicco esercitava su questo mondo di collaboratori una vigilanza occhiuta, testimoniata da libri di conto di ogni tipo e da una fittissima corrispondenza ${ }^{14}$.

Anche i baroni di Roma ricorrevano in genere a forme di gestione diretta basate su un funzionariato strettamente sorvegliato. Il controllo di solito era favorito da periodiche residenze dei signori all'interno dei centri da loro dominati e affidati all'ufficiale locale; oppure avveniva convocando a Roma l'amministratore ${ }^{15}$. Il sistema gestionale affidato a funzionari poteva tuttavia funzionare anche a grande distanza. Il caso limite è quello degli Orsini del ramo di Marino, signori di una decina di castelli. Dal 1320 circa, a capo della famiglia v'era un cardinale, Napoleone, costretto a risiedere ad Avignone, distante un migliaio di chilometri. Eppure anche da remoto si sforzava, con buon successo, di gestire in modo il più possibile diretto la sua signoria. Aveva nominato suo vicario generale a Roma («vicarius in Romanis partibus super negotiis domini») Matteuccio di Poggio, un cadetto di una prolifica famiglia di signori delle montagne umbre: un personaggio strettamente legato al cardinale, che gli inviava dettagliatissimi memoriali zeppi di istruzioni. Quello del 1334, ad esempio, è scritto su sei pergamene. Vi prescriveva di tutto: il periodo migliore per vendere il grano e il vino, le manutenzioni da effettuare sugli edifici, il foraggio da dare ai cavalli, il tipo di moneta con cui riscuotere gli affitti, le istruzioni per l'uso delle quattro casseforti presenti nella rocca signorile (chi ne doveva conservare le chiavi e quali tipo di entrate versare in ciascuna cassaforte), i tempi e le modalità di riscossione dei crediti, l'obbli-

${ }^{14}$ Covini, Potere, ricchezza.

15 Carocci, Baroni di Roma. 
go di chiedere il parere del cardinale prima di intraprendere qualsiasi lavoro straordinario e di fare inviti nella rocca del castello (nessuna eccezione era ammessa, neanche per visitatori importanti, cioè «de familia pape sive regis sive cardinalis», che però potevano venire ospitati e nutriti in altre case di Marino). Il cardinale metteva bocca su tutto: le spese da fare per i corrieri diretti ad Avignone, la concessione di grazie giudiziarie e la remissione di condanne pecuniarie, gli ospiti da accogliere, il tipo di vino che lo stesso vicario era autorizzato a bere (quello locale, e non il più costoso vinum grecum), e tanto altro, compresi i controlli che il vicario doveva esercitare sugli altri ufficiali della signoria. Tutti erano vincolati al rispetto di regole strette, e alla compilazione di conti e rendiconti da inviare a Avignone ${ }^{16}$.

Il sotto idealtipo A2, gestione diretta realizzata tramite ufficiali, nella realtà poteva applicarsi a situazioni molto diverse. Molti piccoli signori si avvalevano della collaborazione di un unico personaggio, che nel Sud e in altre regioni è chiamato balivo (baiulus ${ }^{17}$. Nelle signorie possedute da vasti consorzi familiari, lo stesso ruolo di signore poteva per così dire assumere una fisionomia funzionariale, poiché la guida complessiva della signoria veniva affidata ogni anno a un membro diverso del consortile, eletto dagli altri consorti o scelto a rotazione. Il personaggio designato era responsabile della buona gestione di fronte agli altri membri del consortile, anche se solo in pochi casi compare la richiesta di rendicontazioni dettagliate e scritte. Spesso, del resto, il signore pro tempore doveva utilizzare degli ufficiali scelti dall'insieme dei consorti. Nel 1471, ad esempio, il governo del marchesato piemontese di Bossolasco era ogni anno affidato a un "capitano" membro di una delle quattro famiglie dei consorti, che prima di assumere la carica giurava fedeltà e buona gestione agli altri tre co-signori; per l'amministrazione vera e propria il "capitano" doveva però basarsi su un "vicario" nominato ogni anno congiuntamente da tutti i signori ${ }^{18}$.

Nelle signorie di grandi dimensioni la struttura amministrativa poteva raggiungere una complessità elevata e comportare la redazione di una serie di registri contabili. In Valle d'Aosta, per gestire la loro signoria gli Challant avevano creato una articolata gerarchia di ufficiali, che faceva capo a un ricettore generale, incaricato della supervisione finanziaria, e si poggiava su mistrali, che verificavano l'esecuzione degli ordini signorili, clavigeri addetti a pagamenti e incassi, e soprattutto su castellani, incaricati sia della sorveglianza politica e militare delle fortezze, che della riscossione e della vendita dei prodotti agricoli ${ }^{19}$. Nella signoria del cardinal Napoleone Orsini, in ogni centro v'era un castellanus sive vicarius, un vicecomes, un portarius, uno o due tur-

\footnotetext{
${ }^{16}$ Il memoriale del cardinale è edito in Regesta Chartarum, a cura di Caetani, pp. 87-93; una bella analisi è Cortonesi, Terre e signori, pp. 219-236.

17 Carocci, Signorie di Mezzogiorno, ad indicem.

${ }^{18}$ Una visione d'insieme e il citato esempio di Bossolasco in Fiore, Sistemi parentali e consortili.

19 Del Bo, A proposito della rendita, p. 248.
} 
rerii e dei sergenti. Per quanto ridotti numericamente, questi apparati burocratici comportavano la redazione di una serie di scritture contabili. Quelle degli Challant avevano come modello l'amministrazione dei conti di Savoia, e si incentravano sulle rendicontazioni che i castellani dovevano ogni anno presentare per l'approvazione contabile e la sigla da parte di un cancelliere del signore. Le istruzioni del cardinale Orsini non fanno un elenco completo dei registri di contabilità da redigere, ma menzionano comunque una serie di registrazioni contabili: un libro delle entrate in denaro e in natura («inventarium de omnibus proventibus») da redigere in duplice copia, e da inviare sine mora per controllo alla residenza avignonese del signore; quaderni separati di entrate e spese per ogni castello o grande azienda del cardinale, anch'essi da inviare ad Avignone; un elenco dettagliato dei denari presenti nelle casseforti della rocca signorile; e poi vari altri quaterni di svariata natura e contenuto. A metà del XV secolo, grandi stati signorili come quello di Giovanni Antonio Orsini, principe di Taranto, generavano un volume impressionante di scritture amministrative: per i soli dominii principeschi situati in Terra d'Otranto, ogni anno almeno 1.500 registri di inventari, entrate e uscite e consuntivi erano redatti da ufficiali di vario livello ${ }^{20}$.

Purtroppo queste fonti contabili sono andate quasi tutte perdute. Ci sono delle eccezioni significative, come alcuni rotuli del Friuli redatti da ufficiali signorili (e non dal signore stesso) e i registri di conto degli Challant in Piemonte-Valle d'Aosta, quelli di svariate signorie del Trentino, o frammenti dell'immensa produzione dei dominii del principe di Taranto ${ }^{21}$. Le tipologie documentarie di gran lunga più attestate sono la lista di proventi e il rendiconto $^{22}$. Entrambe mostrano una tendenza crescente alla sorveglianza scritta della gestione. La lista di proventi è la fonte contabile più antica, ma cambia struttura nel corso del XIV secolo: se in origine era solo un elenco dei versamenti teoricamente dovuti dai vari dipendenti di una signoria, dal tardo Duecento inizia sempre più spesso a riportare anche i risultati di inchieste $\mathrm{e}$ i dati desunti da contratti agrari; poi dalla metà del XIV secolo sempre più di frequente aggiunge all'elenco dei proventi teorici l'indicazione di quanto effettivamente incassato ogni anno. Lo scopo di queste registrazioni era quello di migliorare la gestione economica restando in un quadro di grande semplicità contabile e culturale. Manca invece l'interesse a una efficace misurazione della performance economica della signoria, visto che anche nei dominati acquistati da banchieri e mercanti sono assenti non solo libri di conto a partita doppia, ma anche bilanci preventivi e ogni possibilità di effettivi conteggi di avanzi e disavanzi annuali. Quanto alle scritture e alle operazioni di rendiconto, hanno una complessità molto maggiore e richiedono un personale spe-

\footnotetext{
${ }^{20}$ Morelli, Razionalità all'opera, pp. 35-36.

21 Descrizioni delle fonti di amministrazione signorile del Trentino sono in Signorie trentine, a cura di Varanini; Morelli, Razionalità all'opera, in particolare pp. $8 \mathrm{sgg}$., indica le principali iniziative di valorizzazione e edizione delle fonti del principato di Taranto.

22 Buffo, Le scritture della signoria.
} 
cializzato. Assorbono molte risorse, culturali ma soprattutto economiche, e questo spiega la loro relativa rarità. Anche nel loro caso, lo scopo non sembra la misurazione e l'accrescimento della produttività del dominio, quanto piuttosto il desiderio di migliorare l'accountability, cioè la possibilità di accertare l'opera e le responsabilità di quanti collaborano con il signore nella amministrazione.

\section{La gestione delegata (B): problemi di definizione}

Il secondo idealtipo base che mi è sembrato utile definire per modellizzare le forme amministrative delle signorie italiane ha come riferimento principale le pratiche di gestione che non prevedono una partecipazione diretta del signore all'amministrazione, avvenga essa di persona (A1) o tramite ufficiali strettamente controllati (A2). Nelle signorie che possono venire ricondotte all'idealtipo B, vale a dire Gestione delegata, a volte il ruolo gestionale del signore è assente o limitatissimo. In questi casi la delega è totale, e il signore è un puro percettore di rendita. Ma dobbiamo parlare di gestione delegata anche quando l'intervento del signore si manifesta in poche occasioni. Possiamo annoverare nell'idealtipo gestione delegata quei signori che intervengono nella signoria solo al momento di indicare gli amministratori e di riscuotere le rendite, oppure quando si tratta di ricevere suppliche e richieste di grazia dei sottoposti, o di sanare contrasti fra soggetti e ufficiali. La pratica del dominio non si accompagna a un interessamento diretto, costante e ripetuto a breve distanza di tempo circa i modi con cui terre e uomini vengono valorizzati e controllati. Questa lontananza dalle preoccupazioni amministrative a volte è il portato di uno stile di vita che non accorda spazio a operazioni di contabilità e interventi a controllo di fattori e ufficiali vari; altre volte è una scelta volta a massimizzare la rendita, o quantomeno a avere certezza sull'ammontare e la tempistica delle entrate; in altri casi deriva dall'incapacità di scontentare la comunità dei sottoposti, e in particolare quelli più influenti e che svolgono ruoli amministrativi per lunghi periodi, magari in base a una appartenenza familiare.

Formulata questa definizione generale, occorre subito ricordare il carattere astratto delle classificazioni idealtipiche. Nella realtà storica - lo ripeto l'effettiva gestione realizzata da un signore poteva situarsi a metà strada fra due idealtipi. Non pochi signori, inoltre, utilizzavano contemporaneamente forme di gestione che rientrano in più di un idealtipo, ad esempio sorvegliando di persona e con mille cautele l'amministrazione della giustizia e la coltivazione delle riserve, e delegando tramite appalti la riscossione di imposte e censi. In linea generale, nel caso delle signorie che venivano gestite tramite ufficiali, possiamo parlare di gestione diretta se per una qualsiasi ragione il signore conservava un controllo efficace sui propri amministratori (siamo cioè nel sotto idealtipo A2). Per far questo, era opportuno li potesse liberamente scegliere, senza farsi imporre la nomina dall'ereditarietà della funzione o da altre costrizioni; doveva verificarne l'operato; doveva poterli rimuovere a pro- 
pria volontà. Era anche opportuno che gli ufficiali fossero retribuiti con un compenso prefissato e elargito dal signore, e non con una partecipazione agli utili della signoria. La migliore garanzia, infine, si realizzava quando erano personaggi legati soprattutto al signore, estranei alla comunità loro affidata in gestione. Se per l'assenza di questi elementi o per una qualsiasi altra ragione (in primo luogo, il venire meno dell'interesse al controllo gestionale da parte del signore stesso) il livello di autonomia degli ufficiali diveniva così forte da rendere la partecipazione diretta del signore qualcosa che si verificava di rado e con debole capacità di intervento, allora l'amministrazione signorile va piuttosto ricondotta all'idealtipo $\mathrm{B}$, gestione delegata.

Nel concreto della ricerca, come dicevo, spesso risulta difficile distinguere. Esistono dunque sovrapposizioni e rischi di confusione fra l'idealtipo A (soprattutto A2) e l'idealtipo B. Qualche dubbio è suscitato persino dalle pratiche di gestione di signori veramente potenti e interventisti come i baroni di Roma $^{23}$. Infatti in alcune grandi signorie baronali del Lazio del XV secolo, l'autonomia degli ufficiali era così alta che la principale preoccupazione del signore sembra quella di tenerli in qualche modo sotto controllo, stabilendo limiti al loro operato, sollecitando l'invio di proteste dei sudditi contro loro malefatte e cercando di evitare che gli ufficiali accrescessero troppo il proprio potere e i propri prelievi. Nel 1489 l'epistolario di Virginio Orsini cita una massima indicativa: «l'ufficiale che tratta bene i sottoposti è segno che ama il loro signore» («chi tracta bene e' vassalli è singno ama lo Singniore loro») ${ }^{24}$. Come minimo, siamo a cavallo tra gestione diretta e gestione delegata.

Dunque la distinzione fra gestione diretta e gestione delegata spesso è labile. Tuttavia è uno strumento di analisi importante, che è opportuno sia presente allo studioso dei mondi signorili. Inoltre, acquista maggiore valore euristico se articoliamo B, gestione delegata in tre sotto idealtipi. Li illustrerò in base al livello di intervento che richiedono al signore.

\section{La gestione delegata al notabilato locale (B1)}

Il sotto idealtipo B1 fa riferimento a quelle forme di gestione in cui l'amministrazione concreta della signoria è nelle mani di esponenti delle élites locali, cioè di sottoposti ricchi e influenti. A volte, il signore delega completamente tutte le incombenze amministrative; altre volte continua a manifestare qualche interessamento, ma in forme così ridotte e con una capacità di condurre interventi determinanti così bassa da impedire di parlare di gestione diretta.

Questo idealtipo è utile per valutare un gran numero di signorie. Nell'Italia meridionale, ad esempio, dall'inizio del XIII secolo molti signori adottarono un

\footnotetext{
23 Per i secoli XIII-XIV, Carocci, Baroni di Roma; per il secolo XV, Serio, Una gloriosa sconfitta, e Shaw, The political role.

${ }^{24}$ Ibidem, pp. 66-67.
} 
sistema di gestione simile a quello utilizzato dal re nei centri del demanio regio e basato sulla sistematica delega verso elementi di spicco della società rurale ${ }^{25}$. La carica di balivo, l'ufficiale a capo dell'amministrazione signorile, veniva data in appalto ogni anno. Lappaltatore era sempre un abitante della signoria, che comprava l'appalto da solo o associandosi ad altri abitanti. Il prezzo dell'appalto non era regolato dal mercato, ma di fatto fissato dalla consuetudine. Andava pagato parte all'inizio dell'anno, parte in momenti successivi. Chi prendeva l'appalto, si occupava di tutto: della gestione patrimoniale, fiscale e giudiziaria. Faceva amministrare la giustizia e incassava la quota spettante al signore sulle pene inflitte; riscuoteva le imposte sui commerci; riceveva i canoni in natura e in denaro. A volte l'appaltatore si occupava anche della gestione delle riserve signorili, che però in alcuni casi restava sotto il controllo del signore, e in altri era data in appalto a altri abitanti della signoria. Anche i mulini spesso erano dati in appalto a un personaggio diverso dal balivo.

Lappalto o gli appalti a volte assomigliano a una gestione diretta tramite ufficiali, perché il signore conservava buone facoltà di intervento e sorveglianza. Più spesso, però, si traducevano in una completa delega della gestione signorile a membri delle élites locali. Per le signorie di piccole e medie dimensioni questa era l'evenienza più frequente. Di massima il signore continuava a essere una presenza di riferimento e attiva, ma lasciava mano libera a chi aveva acquistato i diritti di gestione. Aveva in tal modo una serie di importanti vantaggi: una contabilità semplificata; pagamenti certi e anticipati; inutilità di grandi apparati di ufficiali; solidarietà dei sottoposti più ricchi e influenti, che venivano cointeressati al buon rendimento di terre, uomini e diritti fiscali. Inoltre questo tipo di amministrazione, che si fondava sulla delega ai notabili locali e sulla loro conoscenza della realtà locale, poteva facilmente venire adottato anche da signori nuovi, che non avevano un radicamento locale. Questa era una condizione molto frequente appunto nel Regno di Sicilia, dove l'avvicendarsi delle dinastie regie, le vicende politico-militari e gli interventi della monarchia determinavano sequestri e riassegnazioni di feudi, cioè frequenti passaggi di mano delle signorie. I numerosi esempi di delega gestionale alle élites rurali offerti dalle regioni centro-settentrionali mostrano poi un ulteriore obbiettivo dei signori: sanare debiti o incassare subito somme di rilievo grazie al pagamento anticipato di un affitto pluriennale. Nelle Prealpi lombarde, nel 1213 sedici abitanti del villaggio di Vallio, signoria del monastero di San Pietro in Monte, presero in fitto per un periodo non specificato ma certamente lungo tutti i redditi e i diritti della signoria, con la sola eccezione di tre tenures contadine; in cambio avrebbero subito pagato la notevole somma di 104 lire a cinque creditori del monastero. Somme cospicue erano spesso versate dai sottoposti anche per la concessione di franchigie che riducevano i prelievi a versamenti fissi annuali, e non a torto si è quindi potuto affermare

${ }^{25}$ Per quanto segue, ampia analisi in Carocci, Signorie di Mezzogiorno, pp. 450-513. 
che «lors d'un affranchissement, la communauté peut en somme devenir fermier des droits seigneuriaux $»^{26}$.

Nella realtà molte forme di gestione possono venire ricondotte a questo sotto idealtipo B1, gestione delegata al notabilato locale. In Friuli, quegli stessi signori che abbiamo visto annotare scrupolosamente censi e redditi, potevano essere costretti a delegare una buona parte delle concrete incombenze amministrative. Questo avveniva di frequente nei villaggi più lontani dal cuore della loro signoria, dove il nobile dominava soltanto un gruppetto di famiglie. In questi casi di debolissima presenza locale del signore, la riscossione di censi e canoni e la sorveglianza dei sottoposti erano in larga misura affidata ai decani, contadini di buona condizione e riconosciuti come capi dal villaggio stesso, che in cambio di una riduzione del proprio canone gestivano i diritti locali del signore ${ }^{27}$. In altre signorie dell'Italia centro-settentrionale, la gestione era delegata non a singoli esponenti di rilievo del mondo locale, ma al comune rurale, che si impegnava a versare ogni anno determinate quantità di denaro e prodotti. In questo caso la delega al notabilato locale non era diretta, ma passava attraverso il controllo del comune. Ad esempio gli accordi stabiliti nel 1233 fra i signori locali è il comune del piccolo castello di Torniella, a sud di Siena, prevedevano la cessione in locazione perpetua al comune stesso di tutte le terre e i beni immobili, dei pascoli, di ogni facoltà di intervento signorile nella nomina dei consoli e nella stessa amministrazione della giustizia, eccetto che per omicidio e tradimento. In cambio i signori avrebbero ogni anno ottenuto dal comune 100 lire, 12 misure di cereali minori e il diritto a ricevere un sussidio di 20 lire in caso di addobbamento di un figlio o matrimonio di una figlia; il solo onere signorile attribuito alle singole famiglie, e non al comune nel suo complesso, era la prestazione di tre corvées l'anno ${ }^{28}$.

Oltre ai vantaggi sopra ricordati, le pratiche amministrative riconducibili all'idealtipo B1 comportavano per i signori anche inconvenienti numerosi e, potenzialmente, esiziali. Dal punto di vista politico-sociale la massiccia delega gestionale alle élites locali diminuiva la capacità di penetrazione locale, rischiando di indebolire o addirittura di distruggere il potere signorile. Non a caso, l'atto di morte di molte signorie fu il passaggio dall'appalto dei diritti signorili alla loro completa vendita in favore di comuni rurali o di appaltatori arricchitisi. Ma anche se non si arrivava a tanto, dal punto di vista economico la delega gestionale al notabilato implicava la rinuncia ad ogni tentativo di innalzare la rendita signorile. Nella Lombardia del tardo Quattrocento, le terre di signori come i Borromeo, che ricorrevano ampiamente alla delega alle società locali, rendevano sette volte di meno, al km², rispetto alle terre di un signore interventista come Cicco Simonetta, di cui ho parlato prima. È bene però non sopravvalutare questa differenza. La rendita elevata delle terre di

26 Menant, Les chartes de franchises, p. 258, con riferimenti anche al caso di Vallio.

27 Le campagne friulane, a cura di Cammarosano, pp. 73-74.

28 Documento edito in Redon, Uomini e comunità, pp. 147-151. 
Cicco era causata in primo luogo dai grandi investimenti produttivi effettuati dal signore e dalla collocazione di Sartirana nella fertile bassa pianura. Per signori come i Borromeo, signori nuovi e che dovevano fronteggiare comunità di sottoposti ricche e dinamiche, delegare alle élites locali gran parte o tutta la gestione della signoria in fin dei conti poteva essere una scelta opportuna non solo dal punto di vista politico, ma anche per potere contare su rendite basse ma certe e non bisognose di investimenti e controlli ${ }^{29}$.

\section{La gestione delegata a professionisti esterni (B2) e la gestione assente (B3)}

Il primo dei due ultimi sotto idealtipi, cioè la gestione delegata a professionisti esterni, fa riferimento a quelle forme di amministrazione con cui la gestione di tutta o di parte della signoria è ceduta a investitori scelti non fra $\mathrm{i}$ dipendenti del signore stesso, ma in un contesto più ampio.

Questa forma di gestione caratterizza soprattutto i secoli dal XV in avanti. Compare peraltro anche nel XIII e XIV secolo, sia pur raramente e soprattutto per alcune signorie ecclesiastiche. In diverse regioni meridionali, ad esempio, dal tardo XIII secolo e soprattutto dalla prima metà del successivo grandi monasteri come la Santissima Trinità di Cava abbandonarono la gestione diretta di molti villaggi posseduti fra Campania, Puglia e Lucania, e iniziarono a conferirli sistematicamente in locazione a personaggi di varia provenienza. Dapprima, nei decenni successivi al divampare della guerra del Vespro nel 1282, gli affittuari furono nobili radicati nei territori circostanti il villaggio, che meglio potevano garantirne la difesa; dalla metà del Trecento, si trattò soprattutto di imprenditori, che risparmiavano a Cava «fatiga e spesa» di gestione $^{30}$. In altre regioni, come la fertile pianura della Lombardia, il ricorso a professionisti esterni nasceva dalla necessità di compiere grossi investimenti richiesti per praticare l'agricoltura intensiva: «gli affittuari della Lombardia si comportano da imprenditori ${ }^{31}$. Agli affittuari potevano essere affidate le stesse migliorie agrarie, e persino la costosa creazione di canalizzazioni per l'irrigazione. Nel 1397 una delle più dinamiche stirpi signorili della Lombardia orientale, i Gambara, cedette per nove anni Gambara stessa e le località vicine a due investitori, che oltre a versare un elevato fitto annuale in denaro, si impegnavano «ad irrigare entro tre anni tutto il territorio a est di Gambara e ad impiantare vigneti in alcune località prestabilite» ${ }^{32}$.

Nelle maggioranza delle altre regioni, più che investitori gli affittuari erano essenzialmente degli intermediari, dotati però di capitali e di specifiche

\footnotetext{
29 Del Tredici, Il profilo economico.

30 Vitolo, Organizzazione dello spazio, pp. 66-69; Tarquini, Le signorie monastiche, pp. 114122; la frase citata nel testo è tratta da un documento del 1478-1480 riportato da Morra, Santissima Trinità di Cava de’ Tirreni, nota 36.

${ }^{1}$ Ago, La feudalità, p. 69.

32 Pagnoni, Gambara, note 32-33.
} 
competenze tecniche e gestionali, e così in grado di garantire al signore un reddito annuale predefinito senza dovere affrontare oneri di amministrazione. Dai primi del XVI secolo, ad esempio, in Piemonte i marchesi del Carretto iniziarono ad «affittare in blocco la maggior parte delle rendite provenienti dalle loro signorie» con contratti di nove anni ${ }^{33}$. Gli esempi sono innumerevoli. Fra le figure più studiate vi sono i cosiddetti "mercanti di campagna" del Lazio moderno. Costoro erano investitori che prendevano in affitto per qualche anno tutta la gestione di una signoria, occupandosi di riscuotere censi e canoni, amministrare la giustizia, valorizzare i pascoli, anticipare capitali per procurare bestiame da lavoro e manodopera per la coltivazione delle riserve. A volte i signori delegavano agli affittuari l'intera conduzione della signoria; altre volte si riservavano parte della gestione. I Borghese ad esempio cedevano ai mercanti di campagna tutta la gestione economica, ma si riservavano la giustizia, il potere signorile dal più alto valore legittimante, che era amministrata dai signori per il tramite di specifici e ben controllati funzionari ${ }^{34}$.

Il successo crescente che questo tipo di gestione signorile ha manifestato in età moderna derivava da molti fattori. I principali furono, da un lato, la sua utilità per signori ormai usi a risiedere stabilmente lontano dalle loro terre; dall'altro lato, e soprattutto, la sua convenienza economica, poiché il professionista esterno aveva i capitali e le conoscenze tecniche necessari per una buona valorizzazione della signoria. Non a caso la documentazione moderna del Lazio attesta numerosi esempi di signori che per qualche anno provarono a gestire direttamente la signoria in modo da incassare tutte le rendite che produceva, salvo poi rinunciare proprio perché le elevate spese di gestione facevano sì che le entrate garantire dall'affitto fossero in fin dei conti superiori a quelle dell'amministrazione diretta ${ }^{35}$.

L'ultimo sotto idealtipo è B3, gestione assente. È un nome strano, un ossimoro, che indica le scelte signorili che di fatto si configurano come una rinuncia completa alla gestione. In questo caso la difficoltà maggiore è quella di distinguere tra azioni che non erano pratiche gestionali, ma mosse legate alla dissoluzione effettiva di ogni potere signorile, e azioni formalmente simili alle precedenti, ma che possono venire annoverate come una forma di gestione in quanto vi si scorge la volontà signorile di non perdere per sempre e in modo totale i propri diritti di dominio, pur rinunciando provvisoriamente, per ragioni politiche o economiche, a ogni controllo effettivo.

Il caso più ovvio erano le signorie oggetto delle più ampie cessioni in feudo. A volte l’infeudazione garantiva al signore una serie di diritti, militari, politici o economici, rendendo difficile parlare di un'effettiva assenza di gestione. Altre volte, invece, con la concessione il signore rinunciava del tutto o in amplissima

\footnotetext{
33 Musso, I del Carretto, pp. 50-51.

34 Forclaz, La famille Borghese, pp. 59-89; Pescosolido, Terra e nobiltà (entrambi i volumi con ampia bibliografia sui "mercanti di campagna").

35 Si vedano ad esempio i tentativi dei Colonna nel XVIII secolo: Armando, Barone, vassalli, pp. 51-52.
} 
parte al controllo della signoria. In questi casi l'ossimoro gestione assente può essere appropriato. La motivazione dell'infeudazione ovviamente era in molti casi politica. Ad esempio, nel 1212 il conte Ildebrandino Aldobrandeschi concesse in feudo perpetuo a dei nobili locali Monteguidi e Montarrenti, senza riservare nulla al proprio controllo. L'infeudazione aveva lo scopo di ampliare la propria clientela e di mantenere un qualche teorico diritto su castelli da poco passati al casato in aree lontane da quelle del suo tradizionale radicamento, $\mathrm{e}$ per questo difficili da amministrare ${ }^{36}$. In altri casi, però, la motivazione era economica: la cessione in feudo o in locazione a lunga durata permetteva di ricevere elevati pagamenti iniziali, e non a caso appare spesso assimilata alla cessione su pegno. Nel 1377, ad esempio, il vescovo di Asti concesse in pegno il castello di Vezza a una famiglia della città, i Da Ponte, in cambio di 8.00o fiorini ${ }^{37}$. Per le grandi signorie ecclesiastiche vi erano poi motivazioni nepotistiche, che spingevano un vescovo o un abate a cedere in feudo o in locazione a lunga durata castelli e signorie. Una parte non piccola delle signorie dei baroni di Roma ha quest'origine ${ }^{38}$. Oppure la motivazione più evidente sembra clientelare e economica: nel 1341 il vescovo di Asti concesse in affitto per dieci anni il castello di Monticello ai Malabayla, una famiglia di banchieri molto legata al vescovo. Il fitto era di 100 lire annue, poi aumentate a 150, che secondo alcuni testi dell'epoca era grosso modo la rendita del castello ${ }^{39}$. Infine, si può parlare di gestione assente anche per i casi in cui una comunità rurale riscattava dal signore tutti i suoi diritti di prelievo e controllo, pur senza formalmente richiedere la fine del dominio signorile. Un esempio remoto è quello della veneta Thiene, i cui vicini ottennero nel 1166 dal signore, il vescovo di Padova, una indipendenza completa, eccetto che per il pagamento di una misura di grano da ogni casa e per il versamento di metà delle multe giudiziarie, peraltro a quel che sembra inflitte dal comune stesso ${ }^{40}$. Sempre nel Veneto, già nella prima metà del XIII secolo si moltiplicano le comunità rurali che, dietro lauti esborsi, riscattavamo dal signore la totalità, o quasi, dei suoi diritti ${ }^{41}$.

\section{Per concludere}

Termina qui la mia illustrazione degli idealtipi che meglio permettono di modellizzare e classificare i caratteri dell'amministrazione delle signorie rurali italiane. Come si è visto dagli esempi di volta in volta avanzati, credo che le ipotesi illustrate nelle pagine precedenti valgano anche per le signorie ecclesiastiche. Queste ultime presentavano naturalmente alcune specificità.

\footnotetext{
36 Collavini, «Honorabilis domus», pp. 301-302.

37 Fresia, I Roero, p. 96, nota 14.

38 Carocci, Baroni di Roma, pp. 97-104.

39 Molino, Monticello d'Alba, pp. 28-30.

40 Documento edito da Castagnetti, Le comunità rurali, pp. 100-101.

41 Un elenco in Castagnetti, Aspetti politici, alle note 82-89.
} 
Le principali erano probabilmente due. La prima nasceva dalla precocità e dall'ampiezza con cui monasteri e, in misura minore, vescovati ricorsero ad affitti e altre forme di gestione delegata a affittuari e imprenditori. Più che dalla volontà di risparmiare a chierici e monaci incombenze gestionali giudicate poco consone alla vita religiosa, questo orientamento derivava dal bisogno di entrate in denaro e invariate negli anni, e dalla convenienza dell'affitto in blocco per gli abati commendatari e quanti altri volevano lucrare sulla concessione di benefici ecclesiastici e per pratiche nepotistiche. La seconda particolarità gestionale dei signori ecclesiastici era una maggiore precocità delle scritture contabili e, per i signori ecclesiastici di grande rilievo, anche una loro maggiore complessità. In parte, questa valutazione può dipendere dalla migliore conservazione degli archivi di monasteri e vescovati, che ci ha trasmesso una grande mole di fonti. Ma in misura molto maggiore sembra un dato reale e inconfutabile, collegato alla migliore preparazione culturale di chierici e monaci e al bisogno di creare una memoria scritta dei diritti della propria comunità che potesse facilmente circolare fra i suoi membri. Questo superiore livello di scritturazione era tanto più necessario in quanto le maggiori istituzioni avevano una struttura articolata di uffici. Già dall'inizio del XIII secolo, ad esempio, il grande cenobio benedettino di Montecassino aveva un'amministrazione centrale con a capo l'abate e articolata in otto mensae distinte, ognuna attribuita a un ufficio maggiore (cellarius, hospitalarius, sacrestarius, ecc.) e dotata ciascuna di specifiche funzioni, di un proprio patrimonio e di un apparato contabile ${ }^{42}$.

Ciascuna forma di gestione aveva specifiche ricadute economiche e sociali. Per l'amministrazione diretta da parte del signore (A1), abbiamo pochi dati economici sicuri, pur se possiamo pensare che permettesse, nella maggioranza dei casi, di massimizzare le entrate signorili grazie alla riduzione dei costi di gestione. Più sicuro è il suo minore impatto sociale. Provvedendo di persona alla gestione del dominio, il signore inevitabilmente limitava il numero di sottoposti che ritraevano risorse materiali e immateriali dalla collaborazione all'amministrazione. Era così depotenziato un fattore che, in altre situazioni, alimentava dinamismo e articolazione della società dominata. La gestione diretta realizzata tramite ufficiali (A2) e quella delegata al notabilato locale (B1) avevano, come vedremo subito, ben maggiori conseguenze sociali.

Nella gestione diretta attraverso ufficiali (A2), i risultati economici potevano cambiare molto. Raggiungevano esiti ottimi nel caso di signori interventisti e ricchi di capitali come Cicco Simonetta, o durante le fasi di più violenta imposizione di nuovi prelievi da parte di signori in ascesa e dei loro collaboratori, come nell'epoca di sviluppo delle facoltà signorili iniziata in Italia centro-settentrionale nell'ultimo ventennio dell'XI secolo e proseguita per almeno due generazioni. Di norma, tuttavia, i dati a disposizione fanno supporre un'efficienza economica bassa, e non a caso per il XII e XIII secolo un

${ }^{42}$ Guiraud, Économie et société, pp. 60-66. 
carattere strutturale delle signorie, allora in gran parte amministrate tramite ufficiali, è stato individuato proprio nell'incapacità culturale e amministrativa di adeguare il prelievo all'andamento della produttività contadina ${ }^{43}$. Anche l'effetto sulla società dominata dell'amministrazione affidata a ufficiali poteva condurre, come avveniva in ambito economico, verso direzioni diverse. Possiamo però essere certi che casi come quello del citato Matteuccio di Poggio a Marino, cioè un ufficiale forestiero le cui fortune o sfortune personali non incidevano in nessun modo sulla fisionomia della società locale, erano minoritari. Nella grande maggioranza dei casi, gli ufficiali signorili erano personaggi locali (come locali erano certamente anche molti ufficiali minori sottoposti allo stesso Matteuccio). La loro partecipazione alla gestione signorile era tutt'altro che irrilevante per le vicende sociali. In modo legale o extra legale, parte delle entrate signorili rimanevano nelle mani degli ufficiali locali e dei loro aiutanti; inoltre la diretta partecipazione alle attività di prelievo e la vicinanza al signore garantivano prestigio e potere, aumentando la possibilità di guadagno e di creazione di clientele. Il servizio al signore in qualità di ufficiali è stato per questo riconosciuto come un fattore formidabile di mobilità sociale e di articolazione delle società contadine ${ }^{44}$.

Fortissimo era anche l'impatto sociale della gestione delegata al notabilato locale (B1). Da un punto di vista economico, questa opzione rappresentava per il signore una scelta per così dire di tranquillità, una rinuncia a cercare di massimizzare la rendita accontentandosi di entrate di ammontare più basso ma sicuro e, soprattutto, conseguito senza alcun fastidio gestionale. Per la comunità dei sottoposti era l'evenienza che lasciava al mondo dominato la maggiore quota di risorse, visto che una parte del prelievo signorile andava ai notabili che lo gestivano. La signoria finiva così per fornire in modo diretto risorse per la preminenza locale. In Italia meridionale, ad esempio, nel XIII secolo proprio le risorse economiche ritratte dall'appalto della gestione signorile furono uno dei fattori più importanti per lo sviluppo e il consolidamento di un ceto di notabili di villaggio, e per le relazioni di clientela che cementavano le società locali 45 .

Per molti aspetti opposta è la valutazione che possiamo dare delle conseguenze economiche e sociali della gestione delegata a professionisti esterni (B2). Anche se i nobili romani di età moderna soffrivano, come abbiamo visto, a veder sparire nelle tasche dei "mercanti di campagna" una bella fetta delle entrate fornite dai loro dominii, affidare la gestione a un professionista non era necessariamente una scelta economicamente irrazionale. Garantiva entrate predefinite e spesso almeno in parte versate in anticipo e, soprattutto, una valorizzazione di terre e sottoposti che si avvantaggiava dei capitali e delle competenze tecniche e imprenditoriali dei professionisti che assumevano la gestione. Non era priva di rischi economici, poiché aveva un costo elevato e il

43 Cammarosano, L'economia italiana.

44 Collavini, Tra campagne e "centri minori", pp. 11-12.

45 Carocci, Signorie di Mezzogiorno, pp. 458-469 e 491-496. 
professionista, reclutato di solito per un breve periodo, tendeva a impoverire le risorse naturali attraverso uno sfruttamento troppo intenso. Per il mondo contadino, questa forma di gestione presentava poche opportunità: era una modalità amministrativa tutta nelle mani di personaggi esterni alla società locale, la quale solo in piccola parte riusciva ad accedere a quei benefici che, come abbiamo visto, ritraeva da altre forme di gestione signorile. Quanto infine a quella che ho etichettato come gestione assente (B3), generalizzare sulle ricadute economiche e sociali è impossibile, tanto ampio appare il ventaglio delle possibilità. Economicamente, poteva essere una scelta utile per i signori bisognosi di realizzare subito somme elevate, sebbene a pregiudizio di futuri redditi. Molto spesso, però, non aveva ragioni economiche, ma politiche, clientelari, nepotistiche, e anche giuridiche (permetteva infatti di aggirare la normativa canonica contro la vendita di beni ecclesiastici). Per la società dominata questa gestione assente a volte si risolveva in un cambiamento del signore di fatto; altre volte segnava un addolcimento sostanziale del dominio, quando era la comunità stessa che assumeva stabilmente la gestione.

Resta infine una domanda importante: possiamo individuare una cronologia e una geografia precisa, cioè una diffusione più o meno ampia di signorie riconducibili ai diversi idealtipi a seconda delle epoche e delle regioni? La risposta più giusta è: sostanzialmente no, non possiamo. Sarebbero necessari ulteriori studi, e una grande articolazione che tenga conto sia delle differenze sub-regionali, sia delle tante tipologie di signoria. Vi sono però alcune tendenze. Come ho detto, signorie riconducibili al sotto idealtipo B2, gestione delegata a professionisti esterni, sono molto presenti nei secoli XV-XVIII, soprattutto nelle signorie di medie e grandi dimensioni. Da parte sua B1, gestione delegata al notabilato locale è onnipresente, in ogni epoca, regione e tipo di signoria, ma appare particolarmente diffusa soprattutto nel Sud e prima del XV secolo, quando deve cedere la supremazia a B2. Quanto ad A1, gestione diretta praticata personalmente dal signore, siamo portati a pensare che fosse tipica soprattutto di una fase remota della signoria, già in diminuzione nel corso del XIII secolo, quando inizia a contraddistinguere solo signori di piccola scala. Il caso dei rotuli tre-quattrocenteschi del Friuli mostra però che forme di direzione personale della amministrazione signorile possono conservarsi o essere istituite anche in epoche tarde. Infine A2, gestione diretta realizzata tramite ufficiali. Per una prima, lunga fase della storia delle signorie italiane, che a volte comprende l'intero medioevo, gli storici tendono spesso a pensare che fosse il tipo di amministrazione più diffusa. Come penso risulti dalle pagine precedenti, credo che la realtà sia più complicata. In ogni caso, appare chiaro che questo tipo di gestione si è accompagnato a un crescente sviluppo di forme di scritturazione, articolazione e gerarchizzazione funzionariale, e infine di accountability, che trovano paralleli con quanto avveniva nelle amministrazioni statali ${ }^{46}$.

\footnotetext{
${ }^{46}$ Per critiche e suggerimenti ringrazio Guido Castelnuovo, Federico Del Tredici, Alessio Fiore e Maria Ginatempo.
} 


\section{Opere citate}

R. Ago, La feudalità in età moderna, Roma 1994.

D. Armando, Baroni, vassalli e governo pontificio. Gli Stati dei Colonna nel Settecento, Roma 2018.

A. Berardozzi, La signoria dell'ospedale di Santo Spirito in Sassia di Roma, in La signoria rurale, a cura di Del Tredici.

P. Buffo, Le scritture della signoria: contabilità e gestione del patrimonio, in La signoria rurale, a cura di Carocci.

Regesta Chartarum. Regesto delle pergamene dell'archivio Caetani, a cura di G. Caetani, II, San Casciano Val di Pesa 1926.

Le campagne friulane nel tardo medioevo: un'analisi dei registri di censi dei grandi proprietari fondiari, a cura di P. Cammarosano, Udine 1985.

P. Cammarosano, L'economia italiana nell'età dei comuni e il 'modo feudale di produzione': una discussione, in «Società e storia», 2 (1979), pp. 495-520.

S. Carocci, Signoria, prelievo rurale, società contadina (sec. XI-XIII): la ricerca italiana, in Pour une anthropologie du prélèvement seigneurial dans les campagnes de l'Occident médiéval, I, Réalités et représentations paysannes, a cura di M. Bourin e P. Martinez Sopena, Paris 2004, pp. 63-81.

S. Carocci, Baroni di Roma. Dominazioni signorili e lignaggi aristocratici nel Duecento e nel primo Trecento, Roma 1993.

S. Carocci, Signorie di Mezzogiorno. Società rurali, poteri aristocratici e monarchia (XII-XIII secolo), Roma 2014.

T. Casini, Le entrate e le risorse materiali dei conti Guidi negli anni '2o del secolo XIII: una stima complessiva, in «Mélanges de l'École française de Rome. Moyen Âge», 132 (2020), pp. 85-105.

A. Castagnetti, Aspetti politici, economici e sociali di chiese e monasteri dall'epoca carolingia alle soglie dell'età moderna, in Chiese e monasteri a Verona, a cura di G. Borelli, Verona 1980, pp. 43-110.

A. Castagnetti, Le comunità rurali dalla soggezione signorile alla giurisdizione del Comune cittadino, Verona 1983.

S. Collavini, "Honorabilis domus et spetiosissimus comitatus». Gli Aldobrandeschi da "conti" a "principi territoriali" (secoli IX-XIII), Pisa 1998.

S. Collavini, Le basi materiali della contea dei conti Guidi tra prelievo signorile e obblighi militari (115o c.-1230 c.), in «Società e storia», 30 (2007),115, pp. 1-32.

S. Collavini, Tra campagne e "centri minori": forme della mobilità sociale nella Toscana rurale del XII secolo, in La mobilità sociale nel Medioevo italiano, 4, Cambiamento economico e dinamiche sociali: secoli XI-XIII, a cura di S. Collavini e G. Petralia, Roma 2019, pp. 1-26.

A. Cortonesi, Terre e signori nel Lazio medievale. Un'economia rurale nei secoli XIII-XIV, Napoli 1988 .

M.N. Covini, Potere, ricchezza e distinzione a Milano nel Quattrocento: nuove ricerche su Cicco Simonetta, Milano 2018.

E. Curzel, Capitolo della cattedrale di Trento, in La signoria rurale, a cura di Del Tredici, pp. $\mathrm{XX}$.

B. Del Bo, A proposito della rendita signorile e delle sue scritture. Le castellanie degli Challant nella Valle d'Aosta (secc. XIV-XV), in La signoria rurale, a cura di Gamberini e Pagnoni, pp. 241-261.

F. Del Tredici, Il profilo economico della signoria lombarda. Il caso dei Visconti e quello dei Borromeo (secoli XIV-XV), in La signoria rurale, a cura di Gamberini e Pagnoni, pp. 19-53.

A. Fiore, Sistemi parentali e consortili nel mondo signorile, in La signoria rurale, a cura di Carocci.

A. Fiore, Il mutamento signorile. Assetti di potere e comunicazione politica nelle campagne dell'Italia centro-settentrionale (1080-113o c.), Firenze 2017 (Reti Medievali E-Book, 29).

B. Forclaz, La famille Borghese et ses fiefs. L’autorité négociée dans l'état pontifical d'ancien régime, Rome 2006.

R. Fresia, I Roero. Una famiglia di uomini d'affari e una terra: le origini medievali di un legame, Cuneo-Alba 1995.

I. Giorgi, Confessione di vassallaggio fatta a Rainone da Sorrento dai suoi vassalli del territorio di Maddaloni, in «Bullettino dell'Istituto storico italiano», 5 (1888), pp. 89-99. 
J.-F. Guiraud, Économie et société autour du Mont-Cassin au XIII e siècle, Montecassino 1999.

F. Lattanzio, Signoria dell'abbazia di S. Paolo fuori le mura di Roma, in La signoria rurale, a cura di Del Tredici.

F. Menant, Les chartes de franchise de l'Italie communale : un tour d'horizon et quelques études de cas, Pour une anthropologie du prélèvement seigneurial dans les campagnes de l'Occident médiéval, I, Réalités et représentations paysannes, a cura di M. Bourin e P. Martinez Sopena, Paris 2004, pp. 239-269.

B. Molino, Monticello d'Alba. Note storiche di un Borgo Medievale, Monticello d'Alba 2008.

S. Morelli, Razionalità all'opera. I bilanci della contea di Soleto nei domini del principe di Taranto Giovanni Antonio Orsini, Napoli 2020.

D. Morra, Santissima Trinità di Cava de' Tirreni, in La signoria rurale, a cura di Del Tredici.

R. Musso, I del Carretto e le Langhe tra medioevo ed età moderna, in «Langhe, Roero, Monferrato. Cultura materiale-Società-Territorio», 6 (2015), 11, pp. 11-83.

L. Orla, Abbazia di San Giusto di Susa, in La signoria rurale, a cura di Del Tredici.

J. Paganelli, Signoria del vescovo di Siena, in La signoria rurale, a cura di Del Tredici.

F. Pagnoni, Gambara, in La signoria rurale, a cura di Del Tredici.

G. Pescosolido, Terra e nobiltà. I Borghese. Secoli XVIII-XIX, Roma 1979.

L. Provero, Forty Years of Rural History for the Italian Middle Ages, in The Rural History of Medieval Europeans Societies. Trends and Perspectives, a cura di I. Alfonso, Turnhout 2007, pp. 141-172.

O. Redon, Uomini e comunità del contado senese nel Duecento, Siena 1982.

A. Serio, Una gloriosa sconfitta: i Colonna tra papato e impero nella prima età moderna (14311530), Roma 2008.

C. Shaw, The political role of the Orsini family from Sixtus IV to Clement VII, Roma 2007.

La signoria rurale nel medioevo italiano, a cura di A. Spicciani e C. Violante, 2 voll., Pisa 19971998.

La signoria rurale nell'Italia del tardo medioevo. Censimento e quadri regionali. Materiali di lavoro, a cura di F. Del Tredici, in corso di pubblicazione.

La signoria rurale nell'Italia del tardo medioevo, 1, Gli spazi economici, a cura di A. Gamberini, F. Pagnoni, Milano-Torino 2019.

La signoria rurale nell'Italia del tardo medioevo, 2, Archivi e poteri feudali nel Mezzogiorno (XIV-XVI sec.), a cura di F. Senatore, Firenze 2021 (Reti Medievali E-Book, 38).

La signoria rurale nell'Italia del tardo medioevo, 3 , L'azione politica locale, a cura di A. Fiore, L. Provero, Firenze 2021 (Reti Medievali E-Book, 39).

La signoria rurale nell'Italia del tardo medioevo, 4, Conclusioni di una ricerca, a cura di S. Carocci, Firenze 2021.

Le signorie trentine nel tardo medioevo, a cura di G.M. Varanini, in corso di pubblicazione.

Strutture e trasformazioni della signoria rurale nei secoli X-XIII, a cura di G. Dilcher, C. Violante, Bologna 1996.

A. Tarquini, Le signorie monastiche nel Regno di Sicilia tra la seconda metà del XIII e il XIV secolo, Tesi di dottorato, Università di Roma Tor Vergata 2018.

G. Vitolo, Organizzazione dello spazio e comuni rurali. San Pietro di Polla nei secoli XI-XV, Salerno 2001.

M. Weber, L'“oggettività” conoscitiva della scienza sociale e della politica sociale [1904], in M. Weber, Saggi sul metodo delle scienze storico-sociali, a cura di P. Rossi, Torino 2001, pp. 187-191.

Sandro Carocci

Università degli Studi di Roma Tor Vergata

carocci@lettere.uniroma2.it 